\title{
Física y Metafísica: notas sobre el espíritu poético de Blake y Whitman
}

\author{
Manuel José BOTERO \\ Universidad Complutense de Madrid \\ manueljosebotero@gmail.com
}

Felizmente, no nos debemos a una sola tradición; podemos aspirar a todas. Mis limitaciones personales y mi curiosidad dejan aquí su testimonio. (Jorge Luis Borges)

Stop this day and night with me and you shall possess the origin of all poems. (Walt Whitman)

Recibido: 13/01/2012

Aceptado: 10/05/2013

\begin{abstract}
RESUMEN
El artículo propone una relación, de alguna manera progresiva, entre la poesía de William Blake y la poesía de Walt Whitman. Por supuesto, no pretende abarcar completamente la poesía de ninguno de los dos autores, sólo centrarse en un aspecto que aparece como un lazo evolutivo entre las dos poéticas: la metafísica. La relación encuentra su base en una misma fuente epistemológica, aquella expuesta por Hegel en su filosofía.
\end{abstract}

Palabras clave: Blake, Whitman, Hegel, metafísica, voz poética, Genio poético.

\section{Physics and Metaphysics: notes on the poetic spirit of Blake and Whitman}

\begin{abstract}
Throughout this article I would like to propose, in sort of a progressive way, a relationship between William Blake's Poetry and Walt Whitman's Poetry. I do not intend, of course, to relate the totality of the works of either poet; I'll just focus on one issue that becomes an evolutive tie between both poetic universes: the metaphysical issue. The relationship finds its base at one same epistemological source, that one exposed by Hegel in his philosophy.
\end{abstract}

Key words: Blake, Whitman, Hegel, metaphysics, poetic voice, Poetic Genius.

SUMARIO: 1. Introducción. 1.1. El idealismo y la percepción. 1.2. La dicotomía. 1.3. La síntesis. 2. Poetic Genius: Blake. 2.1. Reconstrucción filosófica. 2.2. Acerca de eternidad. 2.3. Higher Grounds. 2.4. A manera de conclusión preliminar: $3+4$ casi siempre es igual a 7. 3. Poetic Genius: Whitman. 3.1. 
Brevísima aproximación histórica. 3.2. Reconstrucción filosófica II. 3.3. Acerca de la eternidad II. 3.4. Higher Grounds II. 3.5. A manera de conclusión. $3+4$ debe ser siempre 7

\section{INTRODUCCIÓN}

A lo largo del siguiente artículo quisiera proponer una relación, de alguna manera progresiva, entre la poesía de William Blake (1757-1827), y la poesía de Walt Whitman (1819-1892). Por supuesto, no pretendo abarcar completamente la poesía de ninguno de los dos autores, sólo centrarme en un aspecto que, en mi opinión, conforma un lazo evolutivo entre las dos poéticas: la metafísica. La relación encuentra su base en una misma fuente epistemológica, aquella expuesta por Hegel (1770-1831), en su filosofía.

Este ensayo busca ofrecer un mejor entendimiento de la metafísica (filosofía y religión $)^{1}$ en la poesía de Whitman y no una teoría absoluta acerca su poesía o de la de Blake; tampoco pretende invalidar lecturas alternativas sobre los mismos temas, perspectivas o aproximaciones. Vincent Whitman escribe la disertación doctoral titulada "Clos'd By Your Senses Five": William Blake's Early Illuminated Prophecies and Berkleian Epistemology. La tesis sostiene, entre otras cosas, que la epistemología del filósofo es el bastidor que sostiene las obras proféticas de Blake. Es un trabajo que apunta hacia las posibilidades interpretativas de las obras de Blake; incluso propone que esta relación es esencial para la comprensión de los textos trabajados. No es la dirección que toma este artículo a pesar de que también es dirigido por intuiciones de carácter filosófico y hace, incluso, alguna mención a Berkeley.

Tampoco la relación entre Blake y Whitman es inexplorada; por poner un ejemplo, Sarah Wagstaffe-Ferguson escribe el artículo titulado Points of Contact: Blake and Whitman, que incluye la tumba de Whitman como 'lugar de encuentro' entre los poetas a partir del análisis de los elementos comunes entre las dos poéticas. El punto de partida es el libro de Charles Swinburne titulado William Blake: A Critical Essay.

El vínculo que propongo es más intangible, si se puede, y es que existe un mismo espíritu poético (o filosófico, según se lea) que inunda las expresiones artísticas, tanto de Blake como de Whitman. Aunque no afirma que sea indispensable leer a Blake, o a Hegel en ese caso, para que Whitman sea inteligible, sí es necesario mencionar aquellos caudales de los que es posible que hayan bebido consiente $o$ inconscientemente. Conceptos como el idealismo o la dialéctica, entre otros; o comentar el Poetic Genius, que justifica el atrevimiento de establecerlo como un

\footnotetext{
${ }^{1}$ Utilizo el término metafísica para reunir los dos conceptos, dado que tanto en Blake como en Whitman parecen fundirse en un mismo sentimiento de conexión con lo místico; de igual forma, la poesía y el espíritu poético, comparten esa condición, convirtiéndose así en la piedra angular de las dos poéticas.
} 
sustrato familiar a los dos autores. Tanto es así, que el hecho de que la explicación acerca del Poetic Genius de Blake ${ }^{2}$ sea más extensa, permite que la explicación en Whitman, no sólo sea más corta, sino mucho más clara, convirtiéndola, casi, en un ejercicio de constatación.

No pretendo, en medida alguna, establecer una relación de tipo formal. No he buscado relacionar hechos concretos de la vida real; no por que no los haya sino por que no son esenciales al propósito que persigo. En Interpretación $y$ sobreinterpretación, Umberto Eco sugiere algunas posibilidades acerca de las influencias de los autores: unas directas, otras inconscientes y aquellas que corresponden a la influencia donada por un espíritu común, época o movimiento, que sin atender directa o exclusivamente, a sus fuentes primigenias, permea un modo de pensar y de escribir que pueden dar como resultado unos elementos comunes en dos autores, o literaturas, sin aparente relación formal. Esto es, no es importante si Blake leyó a Hegel, si Whitman leyó a Hegel, si Whitman leyó a Blake. Hay quienes consideran probable que Whitman conociera a Blake a través de Emerson, otros que incluso se atreven a considerar la transmigración de las almas. (En Critical Companion to Walt Whitman: A Literary Reference to His Life and Work, Charles Oliver señala esta conexión en el texto de Swinburne). Está, por supuesto, la opinión que defiende, y acaso prueba, la lectura directa. Lo que parece ser importante, a mi juicio, es la conexión literaria entre los poetas y su conexión con una teoría del conocimiento específica. ${ }^{3}$ Una cuarta posibilidad existe, y es que esta conexión pudiera ser accidental; en realidad sería más poético si así lo fuera.

\subsection{EL IDEALISMO Y LA PERCEPCIÓN}

Hegel se inscribe dentro de la corriente filosófica denominada idealismo, esto es, la vieja doctrina parmenidiano-platónica que advierte que lo 'real' puede ser una apariencia. El argumento metafísico que inventa Parménides, dice Russell, "in one form or another, is to be found in most subsequent metaphysicians down to and including Hegel" (Russell 1972: 48).

Doscientos años antes de Hegel, Descartes había interpretado las pasiones del alma como consecuencia única de reacciones fisiológicas. Por ejemplo, definió el sentimiento denominado amor, como aquella pasión que consiste en desatar encimas gástricas que al introducirse en el flujo sanguíneo ocasiona la aceleración cardiaca. Posteriormente hará su aparición el empirismo, que también se cuenta dentro de las

\footnotetext{
${ }^{2}$ En mi opinión es el mismo que el de Whitman (sólo varía su forma de ser-en-el-mundo: en Blake es objeto, en Whitman sujeto).

${ }^{3}$ La interpretación de la poética de Whitman es extensamente analizada y probada a lo largo de cualquier curso monográfico, de seguir en esa línea, este artículo quedaría convertido en un mero ejercicio de repetición, aspirando, como mayor objetivo, a ser una ampliación de lugares comunes.
} 
doctrinas idealistas, y como el romanticismo pude considerarse como un empirismo de los sentimientos, es conveniente detenerse un momento en esta relación.

El empirismo se enfrenta siempre con el problema de la existencia de la sustancia. Desde Locke hasta Hume, siempre se llega a un punto en donde es necesario hablar acerca de la materia. Poner en tela de juicio el mundo externo aparecería, a primera vista, como una contradicción; teniendo en cuenta que el empirismo es la corriente filosófica que sostiene que todo conocimiento proviene de la experiencia sensible. No obstante, la contradicción se disuelve cuando se entiende que el hecho de que los sentidos perciban alguna impresión no implica que dicha percepción sea adquirida del exterior. Locke, Berkeley y Hume estarán de acuerdo en que no existe ningún elemento lógico que me permita dar cuenta del exterior, sin embargo los dos primeros hacen un esfuerzo por rescatarlo.

John Locke garantiza la existencia del mundo externo con argumentos un tanto tautológicos (Locke 1956: 181). Es necesario saber que la idea es recibida del exterior para saber que ha sido recibida desde el exterior; este argumento, además de cometer una petición de principio, concluye con la duda acerca del proceso mental que se lleva a cabo para la afirmación del postulado. Más adelante alude al sentido común, a la confianza en nuestras facultades, a que el dolor no me engaña, etc., pero sigue sin aportar un proceso mental que garantice esa realidad. Finalmente, la forma como parece salvar el escollo que el mundo le representa, enuncia su teoría de las cualidades secundarias, aquellas percibidas por los sentidos: el olor, el color, el sabor, etc. Estas cualidades secundarias causan impresiones en nuestros sentidos y así nos formamos ideas de ellas. Hasta aquí nada nuevo, siguen siendo percepciones subjetivas, pero dice que las cualidades secundarias necesitan, por fuerza, un soporte, una extensión, sustrato o sustancia, que las sostengan. Así pues, nadie puede percibir un color sin que esté coloreando algo.

Berkeley atacará sin piedad esta postura (Berkeley 1974: 70). Para él también es imposible la existencia de cuerpos externos a la mente que los percibe. Tampoco encuentra una razón válida para garantizar el mundo a partir del raciocinio. La construcción de su argumento es rigurosa. Parte de la afirmación de que ninguna idea existe sin percepción. "Pues la existencia de una idea consiste simplemente en ser percibida" (Berkeley 1974: 69). Continúa diciendo que las ideas impresas y las sensaciones, por complejas que sean, no pueden existir sino en una mente que las perciba. De ese razonamiento se deriva la famosa afirmación de que "ser es ser percibido". Nada puede predicarse de un mundo exterior a una mente que perciba. Aquello que garantiza que el mundo sigue existiendo es que está constantemente percibido por una mente, la mente de Dios. Con esto deja a salvo su postulado que afirma que nada hay fuera de la percepción y deja a salvo el hecho de que haya algo fuera de un individuo en particular.

Hume va a plantear el problema en otro terreno, el terreno del hombre. No niega la existencia del mundo externo y tampoco la afirma. A Hume no parece importarle si existen los cuerpos sensibles: le importa el hombre. Va a interesarse en la causa de la 
creencia en dicho exterior, ni siquiera se detiene en la reflexión acerca de si la creencia es verdadera o por lo menos razonable. Se centra en las razones que llevan a la mente a plantearse la existencia de la materia. Barry Stroud lo explica de manera clara en su libro Hume, (Stroud 1995: 141).

En el Tratado ${ }^{4}$, Hume intenta explicar la conclusión escéptica acerca de si existe o no el mundo externo, en la Investigación ${ }^{5}$, no hay rastro de esa explicación extensa y complicada. Sin embargo, en la Investigación, retoma la preocupación filosófica acerca del mundo externo y llega a la conclusión escéptica como consecuencia inevitable del razonamiento acerca de la materia. Pero en el Tratado, sí cree poder explicar la creencia en ese mundo; Stroud indica de qué manera, (Stroud 1995:142) ${ }^{6}$. La afirmación de Stroud deja a Hume en una posición muy conveniente considerando que la conclusión lógica acerca del mundo se deja a la imaginación: “...nunca daremos realmente un paso fuera de nosotros mismos". (Hume 1984: 169).

En el siglo XX, J.J. Smart habría de proponer que los sentimientos son reacciones químicas ocasionadas en el cerebro por estímulo de los sentidos. Esto es, para conservar el ejemplo, que lo que llamamos amor está en cierta neurona ( $Z$ 108) y que cuando esta es estimulada, reacciona químicamente ocasionando aquello que llamamos amor. La reducción al absurdo de esta línea de pensamiento nace como una objeción al escepticismo epistemológico y fue enunciada por Jonathan Dancy en su Introducción a la epistemología contemporánea. La teoría, popularizada por Hilary Putnam, se conoce como la del "cerebro en una cubeta". Esta perturbadora hipótesis sostiene que si uno no fuera más que un cerebro conectado a un ordenador y si éste fuera muy preciso, no habría forma de saber que no poseemos ni cuerpo ni relación con un entorno, que las experiencias 'vividas' no sería distinguibles de una alternativa 'real', dado que no existe manera de comparar las dos situaciones.

\subsection{LA DICOTOMÍA}

Al hombre contemporáneo se le ha enseñado desde niño cómo el sentimiento y el pensamiento se oponen de manera tan radical, que inevitablemente para que uno de los dos exista, el otro debe desaparecer. Se le ha dicho que los animales actúan por

\footnotetext{
${ }^{4}$ A Treatise of Human Nature (1739-1740).

${ }^{5}$ Enquiry Concerning the Human Understanding (1748).

${ }^{6}$ Los sentidos no pueden proporcionar la percepción de la existencia continua, pues sería necesario que se percibiera algo existente cuando no sea percibido. Lo máximo que nos ofrecen los sentidos es la garantía de una existencia distinta. La razón tampoco genera la idea de la existencia continua de los cuerpos externos, puesto que no hay razonamiento alguno que lleve de la representación de algo a ese algo que se está representando. Hay una conjunción de las dos cosas, pero sólo puede inferirse a partir de la relación de causa y efecto; pero como lo único que puede estar presente en la mente son percepciones, es imposible advertir una conjunción entre una percepción y algo que no es una percepción.
} 
instinto y los hombres (animales racionales) no. También ha aprendido que la comunicación con lo sagrado, y las expresiones de religiosidad, están del todo en contradicción con la racionalidad. Ha aprendido, tristemente, a escoger entre la mística y la razón.

Cuando apareció Darwin cometimos ese error. Lo recibimos con los brazos abiertos, como a Huxley y a Freud, deshaciéndonos en sonrisas. Luego descubrimos que no era posible conciliar las teorías de Darwin con nuestras religiones, o por lo menos así lo pensamos. Fuimos unos estúpidos. Quisimos derribar a Darwin, Huxley y Freud. Pero eran inconmovibles. Y entonces, como unos idiotas, intentamos destruir la religión. Lo conseguimos. Perdimos nuestra fe y el sentido de la vida. Si el arte no era más que la sublimación deseo frustrado, si la religión no era más que un engaño, ¿para qué la vida? La fe lo explicaba todo. (Bradbury 1979: 89)

Hegel afirma, por el contrario, que el sentimiento y el pensamiento son compatibles, que el sentimiento debe ser reflexionado (actividad de la mente) para ser conocido por el hombre. Conceptos tan abstractos como derecho, moral o religión son pensados y el hombre tiene que reflexionar sobre ellos. El pensamiento piensa sobre contenidos, estos contenidos conforman la conciencia y las formas del contenido, o las formas como se expresa ese contenido, son los sentidos y las intuiciones, entre otros. Hegel establece aquí dos cosas notables, una es que los sentimientos deben ser intervenidos por la razón, deben ser pensados; la otra es que la conciencia está conformada tanto por el pensamiento como por los sentimientos. Una de las formas de expresión que encuentran los contenidos de la conciencia son los sentidos.

El pensamiento, indica Hegel, por lo general no se queda inactivo; a esa actividad del pensamiento la denomina reflexión. La reflexión es una acción del pensamiento que consiste en reflejar el pensamiento sobre sí mismo. Al hacer esta reflexión, modifica el contenido pues ahora es necesario pensar sobre el pensamiento y sobre el hecho de haberlo pensado; el concepto al ser pensado, es un nuevo concepto, que al ser pensado genera un tercero, generando así una lógica dinámica. (Esta dinámica será esencial tanto en Blake como en Whitman). El pensamiento filosófico hegeliano, es muy similar al pensamiento poético de los autores aquí tratados.

Ahora bien: siendo la filosofía un modo peculiar del pensamiento, un modo por el cual el pensamiento se eleva al conocer, y al conocer por medio de conceptos, su pensamiento debe también poseer una diferencia respecto a aquel pensamiento cuya eficacia opera sobre todo lo humano y que realiza por antonomasia la humanidad en lo humano por cuanto se identifica con él y el pensamiento es en sí uno sólo. Esta diferencia nace de que el contenido humano de la conciencia, producto del pensamiento, aparece primeramente, no en forma de pensamiento, sino como sentimiento, intuición y representación, formas que han de ser distinguidas del pensamiento formal. (Hegel 1944: 11) 
El pensamiento entonces, debe reflexionar sobre sus contenidos, con esto se tienen dos condiciones: un contenido previo al pensamiento, que de alguna manera genera el movimiento, o dinamismo, del mismo pensamiento y que ese sustrato son las emociones y percepciones. Esto es, que los sentimientos e intuiciones (incluidas las de tipo religioso) no sólo no están en oposición al pensamiento sino que son base fundamental para el mismo. Esta convicción es el que parece recoger Mircea Eliade en su obra Mito y realidad, cuando le restituye la significación 'original' al concepto de mito; aquel que la sociedad racionalista se ha aplicado en olvidar.

El mito designa una «historia verdadera», y lo que es más, una historia de inapreciable valor, porque es sagrada, ejemplar y significativa [...] el mito tiene $-\mathrm{o}$ ha tenido hasta estos últimos tiempos- «vida», en el sentido de proporcionar modelos a la conducta humana y conferir por eso mismo significación y valor a la existencia. (Eliade1991: 7)

\subsection{LA SÍNTESIS}

Teniendo en claro la actividad dinámica del pensamiento, continúo ahora con lo que es, tal vez, el aporte más significativo de Hegel al pensamiento: la dialéctica (tesis, antítesis, síntesis). Para explicar este concepto me remitiré a la explicación que acerca de él hace Bertrand Russell:

Podemos decir, sin contradicción aparente, que el señor A es un tío; pero si dijéramos que el Universo es un señor, nos meteríamos en dificultades. Un tío es un hombre que tiene un sobrino, y el sobrino es una persona separada del tío; por consiguiente un tío no puede ser el todo de la Realidad [...] Primero decimos «la Realidad es un tío.» Esta es la tesis. Pero la existencia de un tío implica la de un sobrino. Como nada existe realmente con excepción de lo Absoluto y tenemos que admitir la existencia de un sobrino, tenemos que concluir: «lo Absoluto es un sobrino.» Esta es la antítesis. Por hallamos contra esto la misma objeción que contra la afirmación de que lo Absoluto es un tío; por consiguiente, nos vemos arrastrados a la opinión de que lo Absoluto es un todo compuesto de tío y sobrino. Esta es la síntesis. Pero esta síntesis es todavía insatisfactoria, porque un hombre sólo puede ser tío si tiene un hermano o una hermana que tienen un hijo, sobrino de aquel. Desde aquí nos vemos llevados a ensanchar nuestro Universo para incluir el hermano o la hermana, con su mujer o su marido. De esta forma -así se afirma-, podemos ser llevados, por la mera fuerza de la lógica, desde cualquier predicado sugerido de lo Absoluto, a la conclusión final de la dialéctica, llamada la «Idea Absoluta». Durante todo el proceso, hay una suposición subyacente de que nada puede ser realmente verdadero, a menos que se refiera a la Realidad como un todo. (Russell 1999: 350)

La condición dinámica del pensamiento es esencial al concepto dialéctico, pues su movimiento es lo que permite la afirmación hegeliana de que la unión del Ser y del No-Ser, es el Devenir. Tampoco ese Devenir es lo Absoluto, pues es necesario que 
devenga de algo; entonces ese devenir se convierte en tesis y su contrario en síntesis, generando una tercera opción que tampoco será el Absoluto, y así ad infinitum.

\section{POETIC GENIUS: BLAKE}

A lo largo de este apartado quisiera llegar a un mejor entendimiento del Poetic Genius de Blake. Basándome en su poema 'Marriage of Heaven and Hell', pretendo llegar a una mejor comprensión del concepto. Aunque, como he mencionado, esta parte será la más extensa, es indispensable familiarizarse con el Poetic Genius, pues la intención de este artículo no es más que mostrar que aquello de lo que habla Blake es precisamente el mismo algo o alguien que toma la palabra en Whitman. Con esto se busca adjudicarle ciertas características al Poetic Genius, no se pretende definirlo, creo que no es posible, sin embargo, parece ser que las intuiciones, tanto de Blake como de Whitman, no son sólo poéticas, sino filosóficas y teológicas: entonces avanzo hacia la exploración del orden metafísico de ideas que puede encontrarse en la poesía de Blake. ${ }^{7}$

\subsection{RECONSTRUCCIÓN FILOSÓFICA}

Without Contraries is no progression. Attraction and Repulsion, Reason and Energy, Love and Hate, are necessary to Human existence. From this contraries spring what the religious call Good \& Evil. Good is the passive that obeys Reason. Evil is the active springing from energy. (Blake 1980: 412)

Blake parece basarse en el argumento expuesto por Hegel: la necesidad de los contrarios para que exista movimiento. En este punto Hegel se distancia, definitivamente de la creencia de Parménides, también compartida por Spinoza, y su teoría recuerda la postura sostenida por Heráclito, quien postula, no sólo que todo está en movimiento y sometido a constante cambio, sino que el Todo es la unión de los opuestos; en Parménides esto es imposible puesto que no existen los opuestos: citando el ejemplo que menciona Russell (1972: 48), para Parménides lo frío es lo nocaliente. Hegel propone la existencia de la Totalidad como un conjunto: un sistema complejo. Blake parece heredar esta convicción.

\footnotetext{
${ }^{7}$ En Origins of Genius: Darwinian Perspectives on Creativity, Simonton ofrece una antropología del concepto de genio que está en las antípodas de la concepción visionaria de Blake y recoge las bases científicas y psicológicas que casan con las premisas darwinistas (Simonton 1999). También, refiriéndose a la imagen romántica de autor, Christine Haynes, en el artículo "Reassessing "Genius" in Studies of Authorship", equipara el genio con el reconocimiento de la autoría y autoridad creativa (Haynes 2005).
} 
Simplificando un poco la teoría, se tiene que en un mundo de "As" la "A" no se reconocería a sí misma, pues la definición de la identidad en Hegel se da a partir de la diferenciación opuesta. Esto es, lo que define a "A" es su conjunto de diferencias internas con "B". "A" es, a su vez, diferente de "C" por el conjunto de sus diferencias internas. Si se tiene que "B" y "C" son diferentes, entonces los dos conjuntos de diferencias $(A \neq B)$ y $(A \neq C)$, serán diferentes dando como resultado " $A$ " $\neq$ " $A$ ". " $A$ " ha cambiado, pues se ha sometido al proceso dialéctico, en el cual la síntesis es un producto diferente de la tesis y de la antítesis. Sin embargo, no se puede presentar la síntesis como una negación de las dos formulaciones precedentes; es la conjunción de las dos lo que permite ese nuevo resultado. "A" no es la negación de "B", ni al contrario, "A" es todo aquello que no es "B", "C", "D", etc. Hasta llegar a ser, incluso, todo aquello que no es " $\mathrm{A}$ ". La dinámica permite que el mundo no sea estático e irreconocible. La síntesis, a su vez, se convierte ya sea en tesis o antítesis, comenzando el ciclo una vez más, resultando en un continuo mundo de ideas en movimiento. El Poetic Genius debe compartir características tanto de la tesis como de la antítesis. De no ser así, sería negado por la síntesis, como se ha visto, o sería estático y, siendo estéril, no se reconocería a sí mismo como una idea, cosa o emoción independiente, clara y distinta.

Siguiendo las leyes de no contradicción, se deriva que nemo dat quod non habet. Aquello que no se mueve, por lo tanto, no puede producir movimiento. Sólo el motor inmóvil, primer principio, que es Dios, podría hacerlo. (Tal vez Blake no estuviera muy conforme si se equiparara al Poetic Genius con Dios -de la tradición judeocristiana-). Pero no solamente debe compartir las características previas, debe, también, compartir las características de la síntesis, pues tiene que tener movimiento sin alterar el conjunto de sus diferencias internas con el mundo no-poético. De no ser así, el Poetic Genius, no sería parte de la síntesis y esta, a su vez, sería algo diferente, privando al Poetic Genius de existencia. Entonces el Poetic Genius debe tener movimiento pero no cambio, lo que conduce a otro tema: El Tiempo.

\subsection{ACERCA DE LA ETERNIDAD}

Eternity is in love with the productions of time [...]

The cistern contains, the fountain overflows [...]

Expect poison from the standing water. (Blake 1980: 416-418)

Parménides definirá el Ser como aquello que es ingénito, inmortal, inmutable y simple. Se ha visto cómo Hegel se aparta de la teoría en lo relativo a lo simple, pero ahora se verá cómo, también, se distancia del concepto de la inmutabilidad del Ser para acercarse, de nuevo, a Heráclito: "nadie se baña dos veces en el mismo río". Una vez más puede reconocerse a Hegel en Blake. 
El tiempo puede entenderse como la generación y continua corrupción de la materia. Aquello que está sujeto al cambio no puede ser eterno pues cambio significa alteración de estado en el tiempo. Blake no parece esperar que su Poetic Genius comparta el concepto de eternidad estática; en la eternidad no se deben encontrar contrarios para prevenir progresión o dinamismo. La eternidad debe ser estática y, por tanto, irreconocible, siempre igual a sí misma, sin cambio ni comparación (tiempo) que pueda ocasionar diferencias ni movimiento. El tiempo es la fuente, la eternidad la cisterna. El tiempo es indispensable para el desarrollo de la obra de arte. La música, por ejemplo, necesita del tiempo para suceder. La poesía necesita del tiempo para hilar las palabras. En la eternidad la poesía no existe; en la fuente de continua corriente, el agua es fresca, nueva y limpia. En la cisterna de la eternidad, el agua no se mueve, es vieja, insalubre, sucia. Con esto se tiene que el cambio es saludable y lleno de vida (sujeto al tiempo) y la eternidad es muerte y esterilidad. ¿Hacia dónde quiere conducir Blake al lector? ¿Cuál es su interés particular en mostrar que lo eterno no es sano? (al menos por sí mismo), si a primera vista todo el mundo aceptaría que lo temporal es corruptible y lo eterno es perfecto? Pienso, tal vez, que la intención radica en poner fin a los prejuicios religiosos. La eternidad es sagrada. Blake procura una unión entre el cielo y el infierno, está tratando de destruir lo que considera meras ideas de dualidad y de contradicción. Trata, al definir el Poetic Genius, de mostrar que los contrarios generan movimiento, que se necesitan mutuamente y que al final se convierten en uno, que son sintetizados en un mismo producto, que llega un punto en el que no se puede escoger cual es bueno y cual es malo, pues están contenidos en uno dentro del otro.

\subsection{HIGHER GROUNDS}

En algún momento de la historia la religión eliminó una instancia del ser. Lo que antes era una tridimensionalidad conformada por el cuerpo, el alma y el espíritu, de pronto se vio convertida en una dualidad cuerpo-alma: principio de animación y sustrato animado. Todo aquello que fue considerado 'malo' del alma se le donó al cuerpo y aquello 'bueno' al espíritu, que heredó el nombre de alma. Esto se debió, en gran medida, a la necesidad de hacer de la religión algo intelectivo, filosófico. La platonización de la religión vía san Agustín en el siglo IV o la aristotelización de la religión vía santo Tomás en el XIII, contribuyen de forma significativa a esta concepción del hombre. Sin embargo, el mismo san Agustín, Juan de la Cruz o sor Juana Inés, en repetidas ocasiones, hacen esfuerzos por acercarse a esa realidad oculta, a esa tercera dimensión del ser. Incluso llega, alguno de ellos, a llamarla 'alma espiritual'.

Blake parece proceder por el mismo camino. Tal vez no de manera tan piadosa como los autores mencionados, pero sí con la misma intuición metafísica de que la 
religión ha dejado algo por fuera. Algo que es necesario buscar y asimilar de manera inmediata para el verdadero desarrollo del ser.

Prisons are built with stones of Law,

Brothels with bricks of Religion. (Blake 1980: 416)

Blake sugiere que los opuestos sólo se encuentran en el mundo de las ideas, esto es, en el mundo del razonamiento intelectual. La religión hace un buen trabajo en esa separación: bien-mal, cuerpo-alma, cielo-infierno, etc. No sólo los separa, sino que elige cuales son positivos y cuales no. En el intento de alcanzar lo que está bien, la religión deja de lado la mitad de ese mundo de ideas por fuera de su control. Eso produce un agujero en la vida del hombre, en el pensamiento, obligándolo a indagar por su cuenta esa mitad perdida de sí mismo. Una idea que desde luego recuerda a Stevenson: un reconocimiento de la oscuridad que completa al hombre. En el caso de Dr. Jekyll el propósito es destruirla o separarla de manera definitiva del ser; en el caso de Blake es abrazarla para completar el ser.

These two classes of men are always upon earth,

$\&$ they should be enemies; whoever tries to reconcile them

seeks to destroy existence.

Religion is an endeavour to reconcile the two.

Note: Jesus Christ did not wish to unite but separate them, as in the Parable of sheep and goats! (Blake 1980: 428)

Si por el contrario, en vez de segregar lo bueno de lo malo, la religión opta por la reconciliación, persiste su carácter destructivo; puesto que si triunfa en su intento de congeniar los enemigos, entonces no se encontrarían deferencias entre ellos y eso detendría el movimiento: el mundo estático y reconciliado sería tan estéril como la cisterna; sería venenoso. La religión busca la eternidad. Jesucristo vino a separar las ovejas de las cabras, El busca que el dinamismo continúe, es por eso que El es el Dador de Vida. Blake acusa a la religión de haber malinterpretado ese hecho, como explica en su conversación con Ezequiel.

Then Ezequiel said. The philosophy of the east taught the first principles of human perception. Some nations held one principle for the origin \& some another, we of Israel taught that the Poetic Genius (as you now call it) was the first principle and all the others merely derivative, which was the cause of our despising the Priests and Philosophers of other countries, and prophecying that all Gods would at last be proved to be originated in our \& to be the tributaries of the Poetic Genius, it was this that our great poet King David desired so fervently \& invokes so patheticly, saying by this he conquers enemies \& governs kingdoms. (Blake 1980: 422-424) 
El Poetic Genius es el Primer Principio: El Movimiento, motor inmóvil. Pero la religión lo ha diseccionado o, al menos, lo ha intentado. El resultado de separar lo que no puede separarse, de desatar lo que no puede ser desatado, es un mundo prosaico con nada más que una fe laica y una desesperanza que ahoga al hombre en un miedo irredimible a la vida que se desenvuelve entre contrarios creados en el mundo de las ideas: vive en el miedo de volver a reunir las piezas que componen al Poetic Genius. Idea que recuerda la referencia bíblica (Ezequiel 6:6) tan bellamente utilizada con posterioridad por Eliot en "The Waste Land". (Eliot 1983: 63)

\subsection{A MANERA DE CONCLUSIÓN PRELIMINAR: $3+4$ CASI SIEMPRE ES IGUAL A 7.}

Messiah or Satan or Tempter was formerly

Thought to be one of the Antediluvians

who are our Energies. (Blake 1980: 428)

Alcanzar una definición exacta del Poetic Genius es, tal vez, una tarea imposible. Es así porque las definiciones serían distintas dependiendo del orden lógico de ideas que se decida utilizar. Filosofía, teología, poesía, imaginación... todas ellas podrían tener una definición individual; tal vez al reunirlas a lo mejor el resultado sería un mejor entendimiento del concepto. Tiene que compartir la eternidad y la corrupción, tiene que estar sujeto al tiempo y ser inmortal; tiene que ser bien y mal, cuerpo y alma; el primer motor y el último movimiento. Si se reemplazara tesis por cielo y antítesis por infierno, la síntesis sería el Poetic Genius. Se crearía así un ciclo sin fin y $\sin$ principio, pues se ha visto que el Poetic Genius es, a su vez, el creador del cielo y el infierno. El resultado sería un movimiento eterno al reunir los opuestos. El vástago del cielo y el infierno es el Poetic Genius, que teniendo relaciones incestuosas con padre o madre, engendrará al Poetic Genius de nuevo, que en principio era el procreador del cielo y del infierno. ${ }^{8}$

\section{POETIC GENIUS: WHITMAN}

En el artículo titulado Hegel's Phenomenological Dialectic and the Structure Of Whitman's "Song of Myself", Cai Zong-Qi, tras explicar brevemente la construcción del sistema fenomenológico de Hegel y la definición de la Noción pura, sostiene que el poema "Song of Myself" de Whitman es una adaptación, o versión, poética del sistema hegeliano. El estudio establece que el desarrollo del yo whitmaniano es un

\footnotetext{
${ }^{8}$ En All Religions are One Blake se refiere al Poetic Genius por primera vez e identifica el propósito en su sistema poético.
} 
sujeto cósmico idéntico al Espíritu Absoluto de Hegel (Zong-Qi 1987: 317). El artículo es de gran ayuda para establecer las relaciones entre el poema y la filosofía hegeliana y, por supuesto, para confirmar la influencia de Hegel en Whitman. Es mi deseo señalar que esa influencia no es evidente exclusivamente en "Song of Myself", sino que permea toda la poesía de Whitman como sustrato metafísico; no sólo como juego poético sino como cosmovisión y honda creencia espiritual.

\subsection{BREVÍSIMA APROXIMACIÓN HISTÓRICA}

Un autor sólo puede escribir acerca de lo que conoce. Incluso lo que imagina está constituido, ya sea por imágenes conocidas o por su negación; los empiristas aclararon ampliamente los reinos de la imaginación, limitándolos a construcciones arbitrarias de elementos conocidos: recuérdese el ejemplo de Berkeley que se refiere a la Nueva Jerusalén con sus calles de oro. El surrealismo hace un maravilloso trabajo al reunir elementos discordantes. ${ }^{9}$ Es por eso que, aunque este ensayo se refiere a una idea de carácter poético, no puede menos que hacer mención, aunque de manera escueta, a la realidad que circundaba a Whitman con respecto del misticismo.

Whitman estuvo rodeado por una serie de tendencias religiosas, pseudo-religiosas y científico-religiosas, que es imposible ignorar al considerar la metafísica de su poesía. No es relevante a este escrito lo que él personalmente pensaba o creía acerca de ellas, sino aquello que puede rastrearse en sus poemas.

Whitman fue criado en un ambiente cuáquero y teísta, su padre era de la corriente deísta a la que pertenecían importantes figuras representantes de lo neo-americano, como Franklin, Jefferson y Paine. Un deísmo que consideraba el mundo como una gran máquina creada por Dios, quien se había retirado para dejarla andar por su cuenta. Aunque en principio era una tendencia que eludía el materialismo, sólo un paso la distanciaba del materialismo ateo. Esto se debía, principalmente a las corrientes materialistas emergentes con las teorías darwinianas que consideraban como único propósito de estudio el entendimiento de la naturaleza. Las sectas milenaristas y finiseculares abundaban, y doctrinas que se confundían entre lo físico y lo espiritual emergían de las esquinas; era un nuevo mundo y nuevas explicaciones estaban a la orden del día. "Phrenology" y el Mesmerismo fueron dos de las corrientes que más sedujeron al espiritualmente inquieto Whitman. El primero era un sistema que daba cuenta de todos los aspectos del ser humano, desde lo físico hasta lo espiritual. El segundo, se basaba la curación por medio de magnetismo, energía y clarividencia. Esta mezcla de ciencia y religión, aunada a su formación paterna, tienen como forma de expresión la compleja poesía que hace de Whitman un poeta del alma

\footnotetext{
${ }^{9}$ Recuérdese la frase de Breton en la que aconseja a los seguidores de la doctrina situar una máquina de escribir en una mesa de disección.
} 
y un poeta del cuerpo. Sin embargo, la secularización que le tocó en suerte vivir, lo convencieron de que la religión era esencialmente una religión sin Dios; era muy humana, muy terrenal, por lo tanto buscó una forma de conciliar ese universo fragmentado que su tiempo le legó. También, la filosofía alemana causa una profunda impresión en el poeta, volviendo su poesía, además, filosófica.

Whitman did not have much firsthand exposure to European Romantic philosophy but received it through edited collections like Hedge's and through magazine articles that he clipped. Although it was not until 1860's that German philosophers, particularly Hegel, had a profound effect on him, their impact was visible in the early poems as well. (Reynolds 1996: 253)

\subsection{RECONSTRUCCIÓN FILOSÓFICA II}

The most merciful thing in the world [...] is the inability of the human mind to correlate all its contents. We live in a placid island of ignorance in the midst of black seas of infinity, and it was not meant that we should voyage far. The sciences, each straining in its own direction, have hitherto harm us little; but some day the piecing together of dissociated knowledge will open up such terrifying vistas of reality $[\ldots]$ that we shall either go mad from the revelation or flee from the deadly light into the peace and safety of a new dark age. (Lovecraft, 1981:12)

El Poetic Genius del que aquí se ha hablado, intenta esta reconciliación de saberes; tal vez no lo hace de una manera tan pesimista como Lovecraft, pues este perpetúa su dicotomía entre el bien y el mal, sugiriendo un horror de tipo cósmico al desvelar tal entramado. Blake y Whitman intentan, con esa unión, disolver tal oposición. Lo que sí es valioso de la cita de Lovecraft, es la forma como acusa la diversificación del saber y la afirmación de que existe un tejido único e inmenso que desborda lo que el hombre conoce como realidad.

Having studied the new and antique, the Greek and Germanic systems,

Kant having studied and stated, Fitche and Shelling and Hegel,

Stated the lore of Plato, and Socrates greater than Plato,

And greater than Socrates sought and stated, Christ divine having

studied long,

I see reminiscent to-day those Greek and Germanic systems,

See the philosophies all, Christian churches and tenets see,

Yet underneath Socrates clearly see, and underneath Christ the divine

I see,

The dear love [...] (Whitman 1978: 290)

La base de toda metafísica es el amor. Una vez unido el conocimiento, el entramado que descubre la voz poética es el amor, un amor que une toda experiencia, 
todo lo sensible, todo lo inteligible y todo lo cognoscible. Si se tiene en cuenta que lo poético y lo filosófico, lo físico y lo metafísico, se corresponden, entonces el Poetic Genius, que es la voz narradora, se identificará con el amor; parece que la voz poética es el amor. Con esto el lector puede aproximarse más a una definición del Poetic Genius (como concepto) al que el artículo ha hecho referencia.

Do I contradict myself?

Very well then I contradict myself, (I am large, I contain multitudes.) [...]

Failing to fetch me at first keep encouraged,

Missing me one place search another,

I stop somewhere waiting for you. (Whitman 1978: 220-222)

Whitman parece basarse en estos versos, al igual que los de Blake (cf. Reconstrucción filosófica), en el principio activo de Hegel; la necesidad de los contrarios para que haya movimiento. Sin embargo, un cambio fundamental se advierte en los versos de Whitman: la voz poética ya no se está refiriendo a un objeto o concepto que se contradiga, en este caso es la voz poética la que se contradice, asumiendo así una de las primeras características del Poetic Genius al que se refería Blake.

The smallest sprout shows that there is really no death [...]

And ceas'd the moment life appear'd.

All goes onward and outward, nothing collapses,

And to die is different from what any one supposed, and luckier. (Whitman 1978: 96)

Puede ya vislumbrarse cómo aquel concepto al que se refería Blake, va tomando voz propia en Whitman, y cómo se conoce más a sí mismo (aquello que se ha bautizado Poetic Genius) efectúa afirmaciones más atrevidas sobre la falsa dicotomía; en los versos anteriores puede verse la negación de la muerte, parte esencial de dialéctica de la humanidad.

\subsection{ACERCA DE LA ETERNIDAD II}

In this broad earth of ours,

Amid the measureless grossness and the slag,

Enclosed and safe within its central heart,

Nestles the seed of perfection. (Whitman 1978: 18)

La voz poética, anuncia de manera clara, que la perfección yace en un mundo corruptible, que de lo temporal proviene lo perfecto, pues la semilla de dicha 
perfección se anida en la tierra, nuestra tierra. Sin embargo, no yace ahí por la condición temporal del mundo, (tiempo que se ha definido aquí como generación y continua corrupción de la materia), sino que está ahí porque lo temporal es ilusorio, es otro falso dualismo dentro del cual el hombre se desenvuelve.

There was never more inception than there is now,

Nor any more youth or age than there is now,

And will never be more perfection than there is now,

Nor any more heaven or hell than there is now. (Whitman 1978: 88)

Esta afirmación acerca del tiempo puede aclarar un poco la turbia impresión que Blake deja en el lector. El Poetic Genius es móvil pero invariable, se desarrolla en el tiempo pero no cambia. Esa contradicción encuentra su solución de la siguiente manera. La voz poética de Whitman existe en un presente absoluto, no ha sido, no será, simplemente es de forma continua: está siendo; y lo hace mientras es poesía, mientras es sustrato de todo conocimiento y de toda generación: es poesía en su estado más puro. Es un estado utópico como el que refiere Gorman Beauchamp, citando a William Dean Howell, en su artículo titulado Changing Times in Utopia: "An order so just that it cannot be disturbed" (Beauchamp 1998: 219).

Tradicionalmente la concepción de Utopía se refiere un modelo de estado en el que reinan la paz y la armonía "for the utopian theorists, disagreement, disorder, conflict are inherently bad; unity, order, harmony are inherently good [...] One in which social life is so perfectly adjusted that no further systemic change is either desirable or possible:" (Beauchamp 1988: 219). Pero más adelante, indica el autor, se dará un cambio en esta concepción, no en la manera de entender la armonía, sino en dónde entender esta armonía, “...the general shift of the locus of utopia from space to time, from an undiscoverable country to an awaiting future" (Beauchamp 1988: 223). Whitman hace precisamente esto al enunciar su sistema poético, cambia el lugar de esta realización para convertirlo en el tiempo de esta realización. Podría concluirse sobre la voz poética de Whitman, de forma preliminar, aquello que se concluye sobre la Utopía:

In such scenarios [...] a dynamic process terminates in stasis -an ultimate or final stasis [...] Progress allows for no such culmination as this, no final stasis. "You have not the idea of Progress" (citando a Bury) "until you conceive that it is destined to advance indefinitely in the future [...] The idea of Progress triumphs [...] only when it overcomes the illusion of finality." Yet this illusion of finality appears to be an inescapable logical consequence of the assumptions of utopian modeling. (Beauchamp 1998: 223-224).

Whitman resuelve esta encrucijada lógica, encuentra la armonía en el tiempo y no en el espacio, pero más importante, en el tiempo presente, no en el futuro. Armoniza 
el desorden y el conflicto, como parte necesaria de su sistema, y conduce al lector "Not onto the good place but into the good time" (Beauchamp 1998:223).

\subsection{HIGHER GROUNDS II}

Creeds and schools in abeyance,

Retiring back a while sufficed at what they are, but never forgotten, I harbour for good or bad, I permit to speak at every hazard,

Nature without check with original energy. (Whitman 1978: 84)

La referencia de Blake a las energías antediluvianas encuentra eco en estos versos de Whitman; el Mesías o Satanás de aquel en los credos y desuso de este. La búsqueda del poeta, el viaje hacia el peligro, la persecución de esa poesía primordial, que será la verdadera voz poética, pone a los dos en una misma embarcación que pretende acabar con las instituciones religiosas; en ningún caso atentan contra los principios fundamentales, tan sólo contra la secularización y la abyecta obediencia que supone asentir a los obtusos principios de la lógica formal, evitando al ser humano conocer los límites de su propia capacidad.

For is the mystic evolution,

Not the right only justified, but what we call evil also justified. (Whitman 1978: 20)

La voz poética advierte a quien escucha de la limitación de las instituciones religiosas, pues anuncia una unión última de los conceptos opuestos de bien y mal. Adquiere características proféticas, pues le habla al mundo directamente tomando la voz del Poetic Genius y se vuelve casi necesaria la relación con texto de Blake titulado "All Religions are One".

I respect Assyria, China, Teutonia, and the Hebrews,

I adopt each theory, myth, god, and demi-god,

I see that the old accounts, bibles, genealogies, are true, without

exception. (Whitman 1978: 50)

\subsection{A MANERA DE CONCLUSIÓN. 3 + 4 DEBE SER SIEMPRE 7.}

Para terminar, quisiera referirme al poema "Chanting the Square Deific", en donde la voz poética, adquiriendo todas las características del Poetic Genius, habla de manera clara y vivaz. Blake hablaba de cierto espíritu poético y trató de definirlo. Ese espíritu poético habla a través de Whitman, es lo que este ejercicio quiere mostrar, esta afirmación encuentra en este poema, tal vez, su prueba más fehaciente. 
Chanting the square deific, out of the One advancing, out of the sides, Out of the old and new, out of the square entirely divine,

Solid, four-sided, (all the sides needed,) from this side Jehovah am I,

Old Brahm I, and I Saturnius am;

Not Time affects me--I am Time, old, modern as any,

Unpersuadable, relentless, executing righteous judgments,

As the Earth, the Father, the brown old Kronos, with laws,

Aged beyond computation, yet never new, ever with those mighty laws rolling,

Relentless I forgive no man--whoever sins dies--I will have that man's life;

Therefore let none expect mercy--have the seasons, gravitation, the appointed days, mercy? No more have I,

But as the seasons and gravitation, and as all the appointed days that forgive not,

I dispense from this side judgments inexorable without the least remorse. (Whitman 1978: 462)

\section{REFERENCIAS}

Beauchamp, Gorman (1998). Changing Times in Utopia. In Philosophy and Literature, vol. 22, Number 1, pp. 219-230. Baltimore, The John Hopkins University Press.

Berkeley, George (1974). Principios del conocimiento Humano. Buenos Aires, Aguilar.

Blake, William (1980). Marriage of Heaven and Hell. En Poesía Completa. Barcelona, Ediciones 29.

Bradbury, Ray (1979). Crónicas Marcianas. Barcelona, Minotauro.

Dancy, Johnatan (1985). An Introduction to Contemporary Epistemology. Oxford, Blackwell.

Descartes, René (1971). Las Pasiones del Alma. Buenos Aires, Aguilar.

Eco, Umberto (1992). Interpretación y sobreinterpretación. Melbourne, Cambridge University Press.

Eliade, Mircea (1991). Mito y realidad. Barcelona, Labor.

Eliot, T. S. (1983). The Waste Land, en Collected Poems. Londres, Faber \& Faber.

Haynes, Christine (2005). Reassessing "Genius" in Studies of Authorship. In Book History, vol. 8, pp. 287-320. Pennsylvania State University Press.

Hegel, G. W. F. (1944). Enciclopedia de las ciencias filosóficas. Méjico, Fondo de cultura económica.

Hume, David (1984). Tratado de la naturaleza humana. Buenos Aires, Orbis.

Hume, David (1994). Investigación sobre el conocimiento humano. Madrid, Alianza.

Locke, John (1956). Ensayo sobre el entendimiento humano. Méjico, Fondo de cultura económica. 
Lovecraft, Howard P. (1981). The Call of Cthulhu. New York, Scott Meredith Literary Agency.

Oliver, Charles M. (2006). Critical Companion to Walt Whitman: A Literary Reference to His Life and Work. New York, Facts on File, Inc.

Reynolds, David S. (1996). Earth, Body, Soul: Science and Religion. In Walt Whitman's America. A Cultural Biography. New York, Vintage Books.

Russell, Bertrand (1999). Hegel. En Historia de la filosofia occidental. Madrid, Espasa Calpe.

Russell, Bertrand (1972). Heraclitus. In The History of Western Philosophy. New York, Simon \& Schuster.

Russell, Bertrand (1972). Parmenides. In The History of Western Philosophy. New York, Simon \& Schuster.

Santa Biblia / Holy Bible (1960). Versión Reina Valera / King James Version. Nashville, Broadman and Holman.

Simonton, Dean Keith (1999). Origins of Genius: Darwinian Perspectives on Creativity. New York, Oxford University Press.

Stevenson, Robert Louis (1994). The Strange Case of Dr Jekyll and Mr Hyde. London, Penguin Group.

Stroud, Barry (1995). Hume. Méjico, Universidad Nacional Autónoma de Méjico.

Wagstaffe-Ferguson, Sarah (2006). Points of Contact: Blake and Whitman. In Sullen Fires Across the Atlantic: Essays in Transatlantic Romanticism. University of Maryland. (Electronic Publication).

Whitman, Vincent M. (2002). "Clos'd By Your Senses Five”: William Blake's Early Illuminated Prophecies and Berkleian Epistemology. UMI Microfilm 3066266.

Whitman, Walt (1978). Chanting the Square Deific. En Poesia Completa. Barcelona, Ediciones 29.

Whitman, Walt (1978). Song of Myself. En Poesía Completa. Barcelona, Ediciones 29.

Whitman, Walt (1978). Song of the Universal. En Poesia Completa. Barcelona, Ediciones 29.

Whitman, Walt (1978). The Base of All Metaphysics. En Poesía Completa. Barcelona, Ediciones 29.

Whitman, Walt (1978). With Antecedents. En Poesía Completa. Barcelona, Ediciones 29.

Zong-Qi, Cai (1987). Hegel's Phenomenological Dialectic and the Structure Of Whitman's “Song of Myself”. In CLIO, 16:4, pp. 313-329. 